\title{
Electronic properties of zigzag $\mathrm{ZnO}$ nanoribbons with hydrogen and magnesium passivations
}

\author{
J.M. All Abbas ${ }^{\mathrm{a}, \mathrm{b}, *}$, P. Narin ${ }^{\mathrm{a}}$, E. Kutlu , S.B. Lisesivdin ${ }^{\mathrm{a}}$, E. Ozbay ${ }^{\mathrm{d}, \mathrm{e}, \mathrm{f}}$ \\ ${ }^{a}$ Faculty of Science, Department of Physics, Gazi University, Ankara, Turkey \\ ${ }^{\mathrm{b}}$ Republic of IRAQ-Ministry of Education-Directorate of Education in Nineveh, Iraq \\ ${ }^{\mathrm{c}}$ Department of Energy Systems Engineering, Faculty of Engineering and Natural Sciences, Ankara, Ylldrrm Beyazlt University, 06010, Ankara, Turkey \\ ${ }^{\mathrm{d}}$ Nanotechnology Research Center, Bilkent University, Ankara, Turkey \\ ${ }^{\mathrm{e}}$ Department of Physics, Bilkent University, Ankara, Turkey \\ ${ }^{\mathrm{f}}$ Department of Electrical and Electronics Engineering, Bilkent University, Ankara, Turkey
}

\section{A R T I C L E I N F O}

\section{Keywords:}

ZnONr

Passivation

DFT

Electronic properties

\begin{abstract}
A B S T R A C T
In this study, the electronic properties of $\mathrm{ZnO}$ nanoribbons with zigzag edges (ZZnONr) have been investigated with Density Functional Theory (DFT). After a geometric optimization, the electronic band structures, the density of states (DOS) of ZZnONr passivated with Hydrogen $(\mathrm{H})$ and Magnesium $(\mathrm{Mg})$ atoms were calculated ZZnONr. It is shown that the increasing width of ZZnONrs has led to a decrement in energy band gap of the studied structures. While $\mathrm{ZZnONr}$ passivated with $\mathrm{Mg}$ for $\mathrm{Zn}$-rich edge have not been shown a spin dependency, the structure passivated with $\mathrm{Mg}$ for O-rich edge have exhibited spin-dependent band structure. The energetically most stable structures have been determined as $\mathrm{ZZnONr}$ passivated with $\mathrm{Mg}$ for $\mathrm{Zn}$-rich edge. $\mathrm{ZZnONr}$ passivated with $\mathrm{Mg}$ atoms for both edges have a graphene-like band structure especially for 8 and 10 atom width structures and this property of ZZnONrs could be important in terms of the electron transport for ZZnONrs.
\end{abstract}

\section{Introduction}

II-VI compound semiconductors have always been an interesting classification of semiconductors due to their possible applications for over a few decades $[1,2]$. Zinc oxide $(\mathrm{ZnO})$ is a generally n-type semiconductor with a wide band gap $(3.37 \mathrm{eV})$ and a large binding energy of excitons $(60 \mathrm{meV})$ at room temperature [3-8]. ZnO has been successfully investigated in the photonics, optoelectronics, spintronics, and gas sensors applications [9-11]. In addition, $\mathrm{ZnO}$ has been regarded as the most suitable candidate for the next generation of ultraviolet (UV) light emitting diode/laser in place of III-V semiconductors [12,13]. ZnO has been studied in a variety of nanostructures including nanowires [14,15], nanorods [16], nanoribbons (Nrs) [17], nanotubes [18,19], nanosheet and bulk structures [20] for both the experimental and the theoretical investigations. Recently, the stability of the graphitic-like structure of $\mathrm{ZnO}$ has been studied as both experimentally and theoretically [21-23]. Similar to graphene nanoribbons (GNRs), the different nanoribbon structures have been widely investigated. For example, many experimental and theoretical studies on electronic and structural properties of different nanoribbons including BN [24-26], SiC [27] and $\mathrm{ZnO}$ [28-30] have been also reported in details.
It has been theoretically predicted that a single layer $\mathrm{ZnO}$ in the honeycomb structure and its armchair edge nanoribbons (AZnONrs) are nonmagnetic semiconductors [30,31], while zigzag edge $\mathrm{ZnO}$ nanoribbons (ZZnONrs) show ferromagnetic-metallic behavior [20], which disappears when the edges of $\mathrm{ZnO}$ nanoribbons are passivated with hydrogen $(\mathrm{H})$ atoms [32]. Therefore, $\mathrm{H}$ passivated $\mathrm{ZnO}$ nanoribbons can be important in terms of spintronic investigations. To our best knowledge, the electronic properties of nanoribbons could be changed with doping, edge passivation, applied an electric field to perpendicular-axis of nanoribbons. The electronic band gap can be controlled in these ways. The edge passivation which is one of these ways has been widely used in GNRs [33]. Similar to GNR studies, edge passivation could be considered as controlling electronic properties of $\mathrm{ZnO}$. A theoretical study of the electronic and magnetic properties of pure and 3d TMs ( $\mathrm{Mn}, \mathrm{Fe}$, and $\mathrm{Co}$ )-doped $\mathrm{ZnO}$ nanoribbons with armchair and zigzag shaped edges were investigated by Oleg Bovgyra et al. [32].

It is well known that $\mathrm{Mg}$ is experimentally used in $\mathrm{ZnO}$ studies [34]. $\mathrm{Mg}$-doped $\mathrm{ZnO}$ materials have attracted much attention because of their unique UV-luminescent properties based on the radiative recombination of the electron-hole pairs [34]. The bulk $\mathrm{ZnO}$ can be alloyed with $\mathrm{Mg}$ atoms and MgZnO alloy can be obtained the wide bandgap

\footnotetext{
* Corresponding author. Faculty of Science, Department of Physics, Gazi University, Ankara, Turkey.

E-mail address: jangizafandy@gmail.com (J.M. All Abbas).
} 


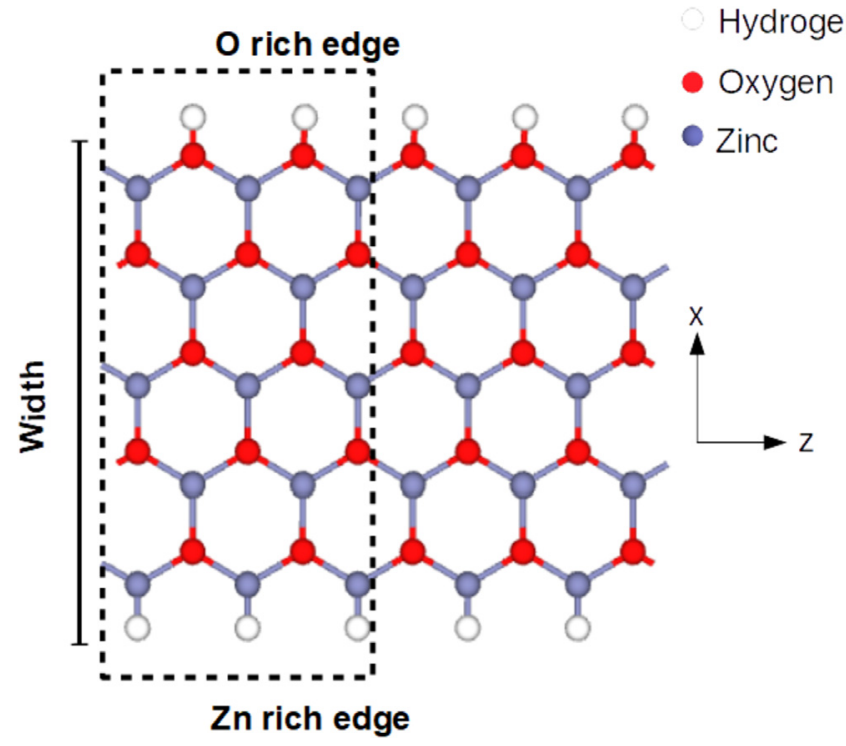

Fig. 1. Atomic configuration of ZZnONrs passivated with $\mathrm{H}$.

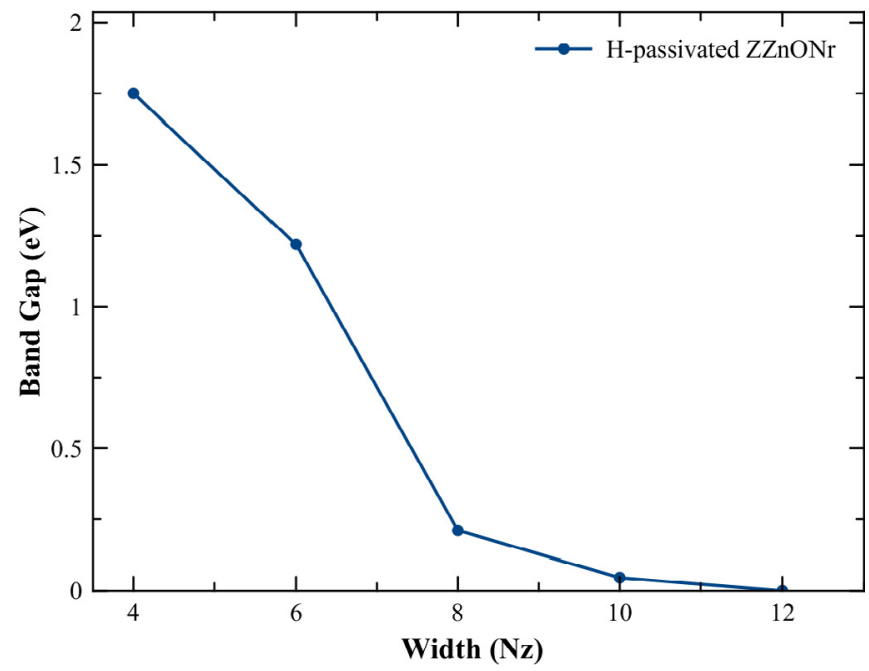

Fig. 2. Electronic band gap values of $\mathrm{ZZnONr}$ with $\mathrm{H}$ atoms as a function of the ribbon width.

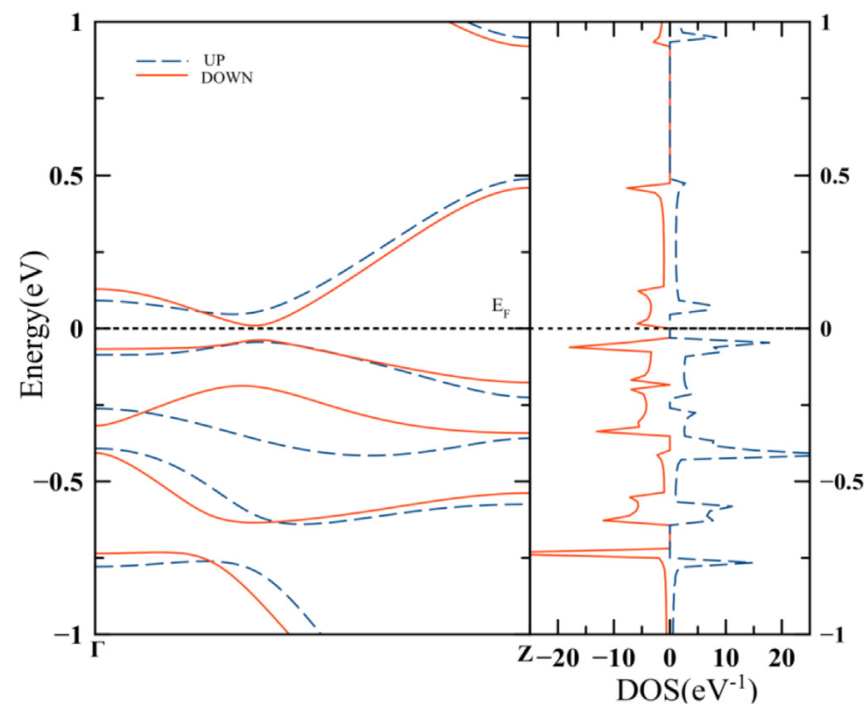

Fig. 3. Electronic band structure and DOS $\mathrm{ZZnONr}$ passivated with $\mathrm{H}$ atoms.

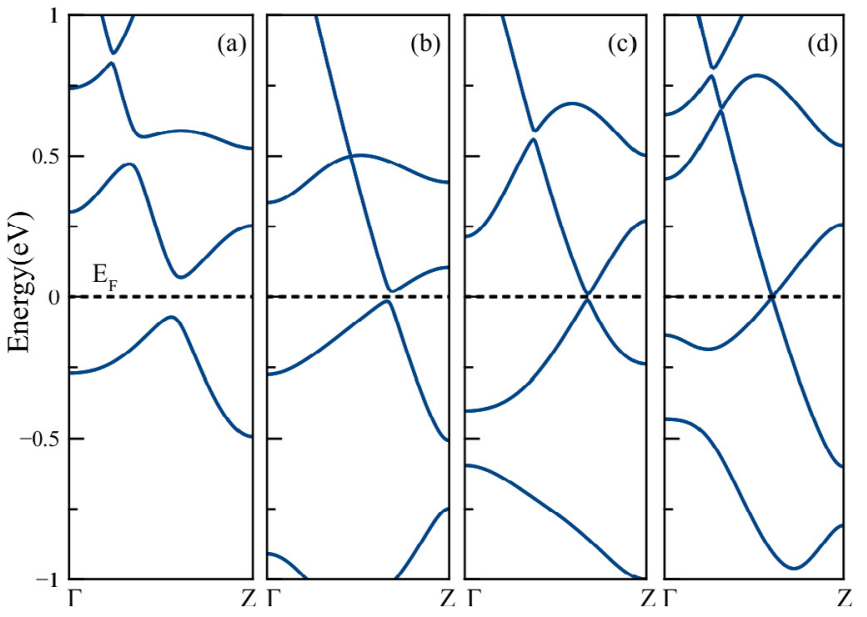

Fig. 4. Electronic band structures of $\mathrm{ZZnONr}$ passivated with $\mathrm{Mg}$ atoms for $\mathrm{Nz}=4-10$ atoms widths.
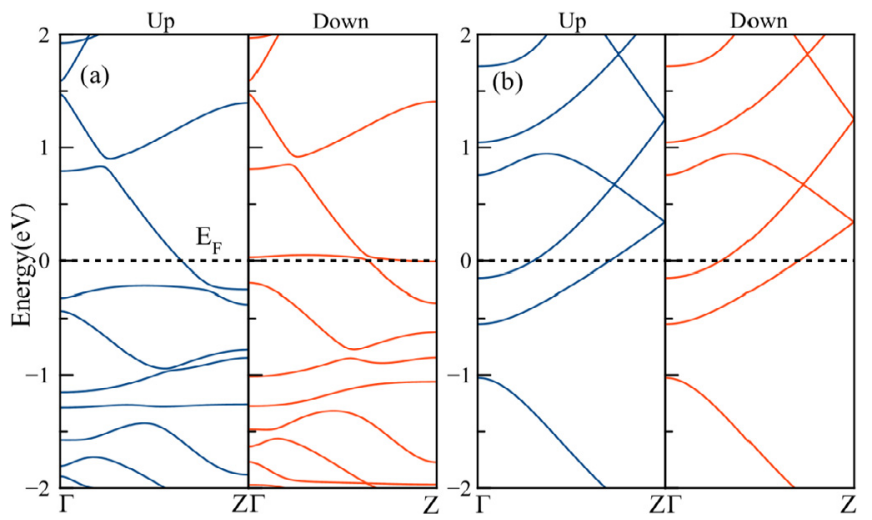

Fig. 5. Electronic band structures for $\mathrm{Nz}=10$ of $\mathrm{ZZnONr}$ with $\mathrm{Mg}$ passivation a) for O-rich edge and b) for $\mathrm{Zn}$-rich edge.

semiconductor material changed between 3.3 and $7.8 \mathrm{eV}$ [34]. However, the effect of $\mathrm{Mg}$ atom on electronic properties of low dimensional $\mathrm{ZnO}$ structures as nanoribbons is still missing.

In this paper, the effect of $\mathrm{H}$ and $\mathrm{Mg}$ atoms on the electronic properties of ZZnONr have been investigated depending on the ribbon width and the edge passivation by DFT calculations.

\section{Numerical methods}

All calculations were investigated for $\mathrm{ZZnONr}$ passivated with $\mathrm{H}$ and $\mathrm{Mg}$ having width $\mathrm{Nz}=4$ to 12 . All calculations were carried out based on DFT by the Atomistix-ToolKit Virtual NanoLab (ATK-VNL) software [35]. Spin-polarized Generalized Gradient Approximation (GGA) in these calculations has been studied as an exchange-correlation potential. The cut-off energy and suitable k-point meshes for the Brillouin zone were used 36.75 Rydberg and $1 \times 1 \times 100$, respectively and the applied force to the nanoribbons was used less than $0.01 \mathrm{eV} / \AA$. The electronic behaviors of $\mathrm{ZZnONr}$ were studied for different ribbon widths and for passivating with $\mathrm{H}$ and $\mathrm{Mg}$ atoms. In Fig. 1 atomic configuration of studied ZZnONr was shown.

The ribbon is periodic in the ' $\mathrm{Z}$ ' direction with the width is illustrate by " $X$ " direction. The electronic band structure, the total and partial DOS (PDOS) structures of $\mathrm{ZZnONr}$ were investigated in details. In order to determine energetically favorable one among the investigated structures, the formation energy $\left(E_{F}\right)$ calculations of each structure have been performed. $E_{F}$ has been calculated using Eq. (1) [36].

$E_{F}=E_{T}+m E_{H}-\left(E_{T, H}+m E_{M g}\right)$ 


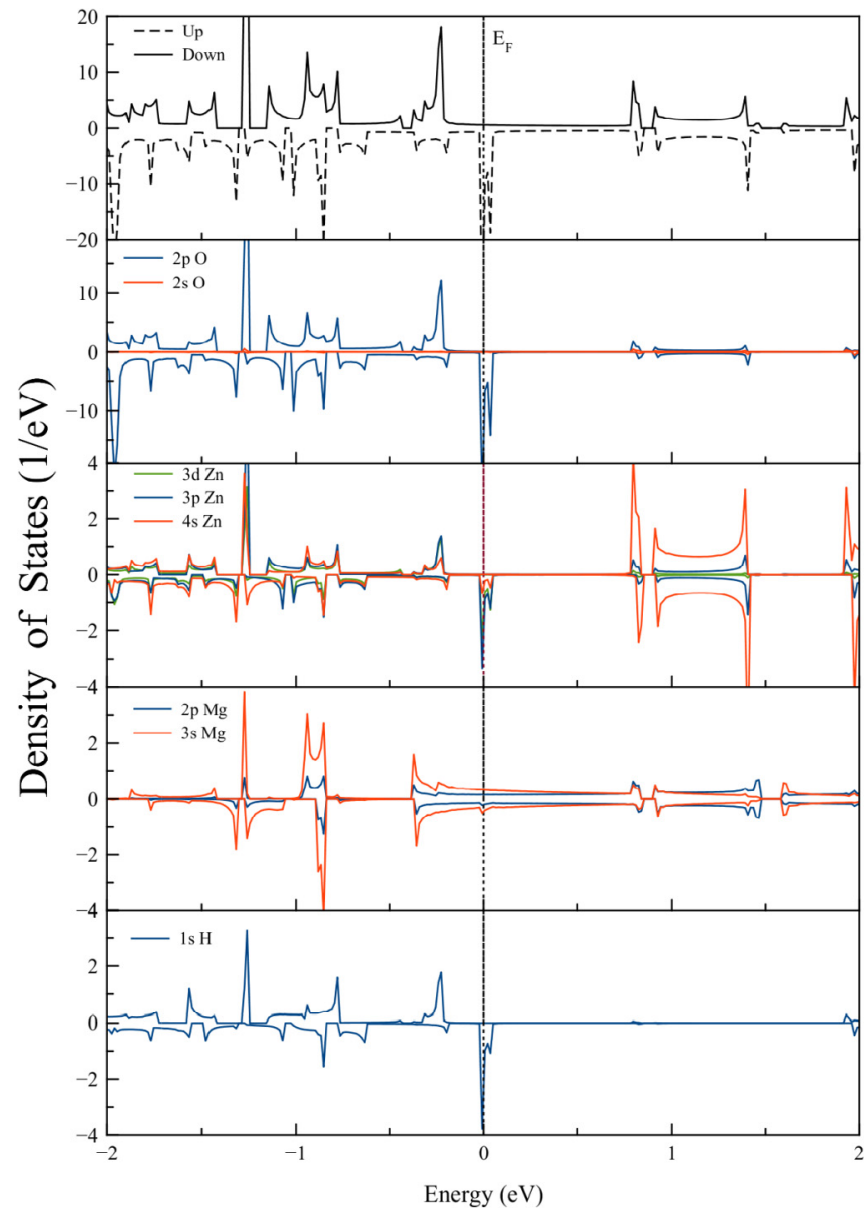

Fig. 6. Total DOS and PDOS of ZZnONr passivated with $\mathrm{Mg}$ for O-rich edge.

where $E_{T}, E_{T, H}, E_{H}$ and $E_{M g}$ are total energies of passivated structures, $\mathrm{H}$ passivated structures, isolated $\mathrm{H}$ and $\mathrm{Mg}$ atoms, respectively. Also $\mathrm{n}$ and $\mathrm{m}$ are number of $\mathrm{H}$ and $\mathrm{Mg}$ atoms.

\section{Results and discussion}

The electronic structures of $\mathrm{ZnO}$ nanoribbon have a semiconductor behavior and can exhibit magnetic property when $\mathrm{ZnO}$ ribbons are passivated with $\mathrm{H}$ [29]. Firstly, ZZnONr passivated with $\mathrm{H}$ atoms for both edges have been studied depending on the width of ZZnONr. The electronic bandgap is decreased with increasing width of $\mathrm{ZZnONr}$ as shown in Fig. 2 similar to Chen et al.'s study [37]. In GNRs, there is a similar behavior between width of nanoribbon and electronic band gap values. Narrow nanoribbons exhibit a larger electronic band gap because of quantum-confinement effects [38]. Also, as increase width of nanoribbons, the interaction between the edges of nanoribbon is decreased and hence this leads to a decrement in band gap of nanoribbon [32].

Experimentally, the different band gap energy has been obtained for different width of $\mathrm{ZnO}$ nanoribbons. For example, $3.32 \mathrm{eV}, 3.26$ and $3.22 \mathrm{eV}$ of band gap have been determined in width of $50-150 \mathrm{~nm}$, $300-500 \mathrm{~nm}$ and $2-3 \mu \mathrm{m}$ of $\mathrm{ZnO}$ nanoribbons, respectively [39-41]. According to experimental results, it is shown that the increasing width of $\mathrm{ZnO}$ nanoribbons have led to increase band gap of $\mathrm{ZnO}$ nanoribbon. In the calculations, the spin-polarized calculations have been performed to investigate the spin dependency as a function of the studied ZZnONr's widths. It is observed that there is no spin dependence in 4, 6 and 8 of the width of ZZnONrs. For 10 atom width of ZZnONr, however, the spin-dependent electronic band structures have been obtained. These results indicate that spin properties of $\mathrm{ZZnONr}$ can be controlled with

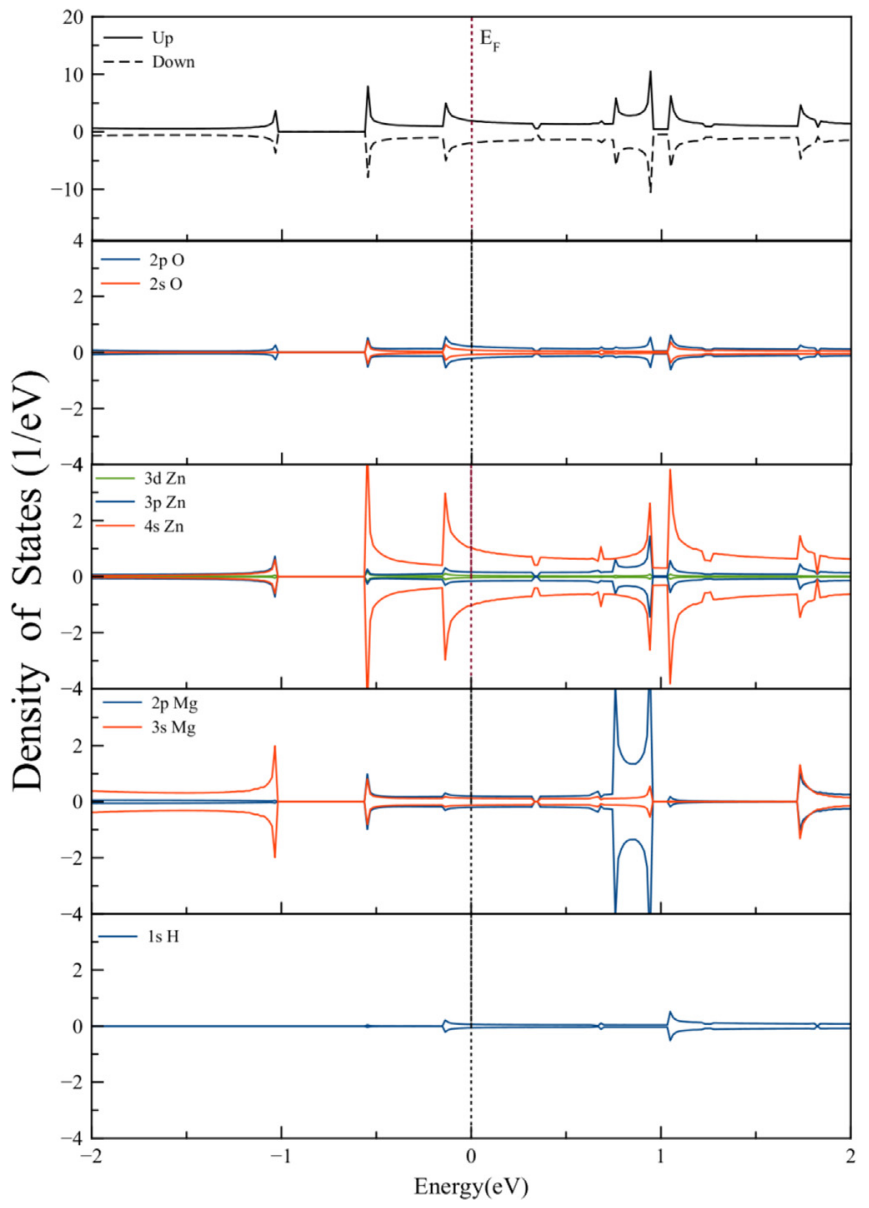

Fig. 7. Total DOS and PDOS of ZZnONr passivated with Mg for Zn-rich edge.

Table 1

Calculated electronic parameters for ZZnONrs passivated with $\mathrm{Mg}$ and $\mathrm{H}$ atoms.

\begin{tabular}{llll}
\hline \#ZZnONr & Formation Energy $(\mathrm{eV})$ & Type of bandgap & $\mathrm{E}_{\text {gap }}(\mathrm{eV})$ \\
\hline Bare & - & - & 0 \\
Both edge $\mathrm{H}$ & - & Direct & 0.460 \\
Both edge $\mathrm{Mg}$ & 3.39 & Direct & 0.006 \\
Zn-rich edge $\mathrm{Mg}$ & -3.38 & - & 0 \\
O-rich edge $\mathrm{Mg}$ & 102 & - & 0 \\
\hline
\end{tabular}

width of nanoribbons. Previously, ZZnONrs have been shown the increasing of spin properties depending on increasing of nanoribbon's width [9]. Furthermore, as seen in Fig. 2 for 12 atom width of $\mathrm{ZZnONr}$, the electronic band gap is closed. The electronic band structure and DOS for $10-\mathrm{ZZnONr}$ passivated with $\mathrm{H}$ are plotted in Fig. 3. The blue and red lines are represented spin up and spin down band structures, respectively. It is shown that the calculated $10-\mathrm{ZZnONr}$ has a direct bandgap semiconductor behavior. The spin down band gap and spin up band gap values are found to be 0.047 and $0.095 \mathrm{eV}$, respectively. Because $10-\mathrm{ZZnONr}$ is the depending on spin properties, it has been investigated more details on the electronic properties of $\mathrm{ZZnONr}$ passivated with $\mathrm{Mg}$ for both edges of the structure.

The electronic band structures of $\mathrm{ZZnONr}$ passivated with $\mathrm{Mg}$ atoms which have the different ribbon widths are shown in Fig. 4. The electronic band gap is narrower and then closed as increases the width of $\mathrm{ZZnONr}$ from 4 to 10 atom width. Similar to $\mathrm{ZZnONr}$ passivated with $\mathrm{H}$, ZZnONrs passivated with Mg have exhibited a similar closing band gap trend as increases the nanoribbon's width. This behavior is expected as shown in previous nanoribbon studies [42]. It is shown that all investigated electronic band structures have a nearly direct band gap. As 
increase width of nanoribbon, the band structures become to show a linear dispersion, unlike conventional parabolic electronic band structure. Also, it is clearly shown that the electronic band structure of ZZnONr passivated with $\mathrm{Mg}$ atoms had 8 and 10 atom width have a band structures graphene-like. Moreover, all studied structures have not been exhibited spin-dependent properties. Contrary to GNRs, the edges of $\mathrm{ZnO}$ nanoribbons are different from each other as one edge is an O-rich edge, other edge is a Zn-rich edge. Therefore, the electronic properties of ZZnONrs are strongly dependent on the edges of ZZnONrs.

Additionally, to determine the effect of $\mathrm{Mg}$ passivation for O-rich and $\mathrm{Zn}$-rich edges on electronic properties $10-\mathrm{ZZnONr}$, the electronic band structure of ZZnONrs passivated with $\mathrm{Mg}$ atoms for only one edge have been calculated and are shown in Fig. 5. As it can be seen from Fig. 5a), the electronic band structure of $\mathrm{ZZnONr}$ passivated with $\mathrm{Mg}$ for O-rich and Zn-rich edges has a metallic behavior. In addition, unlike ZZnONr passivated with $\mathrm{Mg}$ for O-rich edge structure, $\mathrm{ZZnONr}$ passivated with $\mathrm{Mg}$ for $\mathrm{Zn}$-rich edge has not been a spin-dependent band structure.

In order to explain the shell contribution of $\mathrm{Zn}, \mathrm{O}, \mathrm{Mg}$ and $\mathrm{H}$ atoms to the electronic band structure of ZZnONr, total density of states (DOS) and partial DOS (PDOS) calculations have been performed. Fig. 6 shows the spin-dependent total DOS and PDOS of ZZnONr passivated with $\mathrm{Mg}$ for O-rich edge while the valence band is mostly coming from the O-2p shell, the conduction band consists of Zn-4s and O-2p shells both spin up and spin down bands. For the spin up bands, there is a strong density at Fermi level due to the crossing of conduction and valence bands shown in band structure. It is shown that this density is mostly originated from the O-2p shell. Furthermore, H-1s and $\mathrm{Zn}-2 \mathrm{p}$ shells have a lower effect on this density. Fig. 7 shows total DOS and PDOS of ZZnONr passivated with $\mathrm{Mg}$ for Zn-rich edge. As shown in Fig. 7, the $\mathrm{Zn}-4 \mathrm{~s}$ shell is contributed to total DOS at near Fermi level. Mg-2p shell, as well as $\mathrm{Zn}-4 \mathrm{~s}$ shell, has a strong effect on the total DOS about at $1 \mathrm{eV}$. In passivation with $\mathrm{Mg}$ atoms of O-rich edge, the spin property is originated from $\mathrm{O}$ edge of $\mathrm{ZZnONr}$. It is shown that $2 \mathrm{p}$ orbital of $\mathrm{O}$ atoms is determined the spin properties of ZZnONr. A previous study reveals that while $\mathrm{p}$ orbital of $\mathrm{O}$ has a strong spin polarization, $\mathrm{d}$ orbital of $\mathrm{Zn}$ has a weak spin-polarization [32]. Our result shows a good agreement with results of this study. It can be inferred from that to passive the $\mathrm{O}$ rich edge of ZZnONr can be contributed to spin properties of $\mathrm{ZZnONr}$.

$E_{F}$ values and the band gap of investigated structures are listed in Table 1. As it can realize that the structure passivated with $\mathrm{Mg}$ for $\mathrm{Zn}$ rich edge is the most stable structure among passivated structures. In Table I, a negative formation energy value show that $\mathrm{Mg}$ passivation for Zn-rich edge of ZZnONr can be spontaneously formed [43]. Also, the negative formation energy means that investigated structures can be prepared more appropriate in experimental studies [44].

\section{Summary}

In this study, the electronic properties of $\mathrm{ZZnONr}$ have been investigated using first-principles calculations. To explain electronic structures of $\mathrm{ZZnONr}$ passivated with $\mathrm{H}$ and $\mathrm{Mg}$ atoms, the electronic band structures, total and PDOS and the $E_{b}$ for each investigated structure have been calculated. It is shown that the increasing widths of ZZnONrs have led to a decrement in energy band gap of the structures. While ZZnONr passivated with $\mathrm{Mg}$ for Zn-rich edge have not been shown spin dependency, the structure passivated with $\mathrm{Mg}$ for O-rich edge have exhibited spin-dependent band structure. O-rich edge of $\mathrm{ZZnONr}$ has a stronger spin polarized structure. The most stable structures have been determined as the largest width ZZnONr passivated with $\mathrm{Mg}$ for $\mathrm{Zn}$-rich edge. $\mathrm{ZZnONr}$ passivated with $\mathrm{Mg}$ atoms for both edges have a graphene-like band structure especially for 8 and 10 atom width structures and this property could be important in terms of electron transport for ZZnONrs. Spin properties of ZZnONr are strongly dependent on edge structures of ZZnONr as Zn-rich and O-rich edges.

\section{Acknowledgment}

This work was supported by TUBITAK under Project No. 116F197. E.O. acknowledges partial support from the Turkish Academy of Sciences. S. B. L was supported in part by the Distinguished Young Scientist Award of Turkish Academy of Sciences (TUBA-GEBIP 2016).

\section{References}

[1] H. Morkoc, S. Strite, G.B. Gao, M.L. Lin, B. Sverdlov, M. Burns, Large-band-gap SiC, III-V nitride, and II-VI ZnSe-based semiconductor device technologies, J. Appl. Phys. 76 (3) (1994) 1363-1398.

[2] S. Adachi, Properties of Semiconductor Alloys: Group-IV, III-V, and II-VI Semiconductors vol. 28, John Wiley \& Sons, 2009.

[3] D.G. Thomas, The exciton spectrum of zinc oxide, J. Phys. Chem. Solid. 15 (1-2) (1960) 86-96.

[4] V. Srikant, D. R_ Clarke, On the optical band gap of zinc oxide, J. Appl. Phys. 83 (10) (1998) 5447-5451.

[5] A. Teke, Ü. Özgür, S. Doğan, X. Gu, H. Morkoç, B. Nemeth, J. Nause, H.O. Everitt, Excitonic fine structure and recombination dynamics in single-crystalline $\mathrm{ZnO}$, Phys. Rev. B 70 (19) (2004) 195207.

[6] Ü. Özgür, Ya. L. Alivov, C. Liu, A. Teke, M.A. Reshchikov, S. Doğan, V. Avrutin, S. J. Cho, H. Morkoç, A comprehensive review of $\mathrm{ZnO}$ materials and devices, J. Appl. Phys. 98 (4) (2005) 11.

[7] A. Janotti, C.G. Van de Walle, Fundamentals of zinc oxide as a semiconductor, Rep. Prog. Phys. 72 (12) (2009) 126501.

[8] B.K. Meyer, H. Alves, D.M. Hofmann, W. Kriegseis, D. Froster, F. Bertrman, J. Christen, A. Hofmann, M. Straburg, M. Dworzak, U. Haboeck, A.V. Rodina, Bound exciton and donor-acceptor pair recombinations in ZnO, Phys. Status Solidi 241 (2) (2004) 231-260.

[9] R.A. Botello-Méndez, F. Lopez-Urias, M. Terrones, H. Terrones, Magnetic behavior in zinc oxide zigzag nanoribbons, Nano Lett. 8 (6) (2008) 1562-1565.

[10] A.R. Botello-Méndez, F. Lopez-Urias, M. Terrones, H. Terrones, Enhanced ferromagnetism in $\mathrm{ZnO}$ nanoribbons and clusters passivated with sulfur, Nano Res. 1 (5) (2008) 420-426.

[11] Z.L. Wang, Zinc oxide nanostructures: growth, properties and applications, J. Phys. Condens. Matter 16 (25) (2004) R829.

[12] Y. Ryu, T.S. Lee, J.A. lubguban, H.W. White, B.J. Kim, Y.S. park, C.J. Youn, Next generation of oxide photonic devices: ZnO-based ultraviolet light emitting diodes, Appl. Phys. Lett. 88 (24) (2006) 241108.

[13] A. Tsukazaki, A. Ohtomo, T. Onuma, M. Othani, T. Makino, M. Sumiya, H. Ohno, Repeated temperature modulation epitaxy for p-type doping and light-emitting diode based on ZnO, Nat. Mater. 4 (1) (2005) 42.

[14] Q. Wan, Q.H. Li, Y.J. Chen, T.H. Wang, X.L. He, J.P. Li, C.L. Lin, Fabrication and ethanol sensing characteristics of $\mathrm{ZnO}$ nanowire gas sensors, Appl. Phys. Lett. 84 (18) (2004) 3654-3656.

[15] Y.C. Kong, D.P. Yu, B. Zhang, W. Fang, S.Q. Feng, Ultraviolet-emitting ZnO nanowires synthesized by a physical vapor deposition approach, Appl. Phys. Lett. 78 (4) (2001) 407-409.

[16] W.I. Park, D.H. Kim, S.W. Jung, G.C. Yi, Metalorganic vapor-phase epitaxial growth of vertically well-aligned ZnO nanorods, Appl. Phys. Lett. 80 (22) (2002) $4232-4234$.

[17] H. Si, B.C. Pan, Strain-induced semiconducting-metallic transition for ZnO zigzag nanoribbons, J. Appl. Phys. 107 (9) (2010) 094313.

[18] Y.J. Xing, Z.H. Xi, Z.Q. Xue, X.D. Zhang, J.H. Song, R.M. wang, D.P. Yu, Optical properties of the $\mathrm{ZnO}$ nanotubes synthesized via vapor phase growth, Appl. Phys. Lett. 83 (9) (2003) 1689-1691.

[19] A. Fathalian, J. Jalilian, First principle study of unzipped zinc oxide nanotubes, Phys. Lett. 374 (46) (2010) 4695-4699.

[20] G. Qin, X. Wang, J. Zheng, C. Kong, B. Zeng, First-principles investigation of the electronic and magnetic properties of $\mathrm{ZnO}$ nanosheet with intrinsic defects, Comput. Mater. Sci. 81 (2014) 259-263.

[21] C.L. Freeman, F. Claeyssens, N.L. Allan, J.H. Harding, Graphitic nanofilms as precursors to wurtzite films: theory, Phys. Rev. Lett. 96 (6) (2006) 066102.

[22] C.L. Freeman, F. Claeyssens, N.L. Allan, Y. Sun, M.N. Ashfold, J.H. Harding, Growth of ZnO thin films-experiment and theory, J. Mater. Chem. 15 (1) (2005) 139-148.

[23] C. Tusche, H.L. Meyerheim, J. Kirschner, Observation of depolarized ZnO (0001) monolayers: formation of unreconstructed planar sheets, Phys. Rev. Lett. 99 (2) (2007) 026102.

[24] F. Zheng, G. Zhou, Z. Liu, J. Wu, W. Duan, B.L. Gu, S.B. Zhang, Half metallicity along the edge of zigzag boron nitride nanoribbons, Phys. Rev. B 78 (20) (2008) 205415.

[25] W. Chen, Y. Li G. Yu, C.Z. Li, S.B. Zhang, Z. Zhou, Z. Chen, Hydrogenation: a simple approach to realize semiconductor - half-metal - metal transition in boron nitride nanoribbons, J. Am. Chem. Soc. 132 (5) (2010) 1699-1705.

[26] C.H. Park, S.G. Louie, Energy gaps and Stark effect in boron nitride nanoribbons, Nano Lett. 8 (8) (2008) 2200-2203.

[27] L. Sun, Y. Li, Z. Li, Q. Li, Z. Zhou, Z. Chen, J.G. Hou, Electronic structures of SiC nanoribbons, J. Chem. Phys. 129 (17) (2008) 174114.

[28] Y. Wang, B. Wang, Q. Zhang, D. Shi, S. Yunoki, F. Kong, N. Xu, A simple capacitor model and first-principles study of carbon-doped zigzag $\mathrm{ZnO}$ nanoribbons, Solid State Commun. 152 (6) (2012) 534-539.

[29] M. Topsakal, S. Cahangirov, E. Bekaroglu, S. Ciraci, First-principles study of zinc 
oxide honeycomb structures, Phys. Rev. B 80 (23) (2009) 235119.

[30] R.A. Botello-Méndez, M.T. Martinez- Martinez, F. Lopez- Urias, M. Terrones, H. Terrones, Metallic edges in zinc oxide nanoribbons, Chem. Phys. Lett. 448 (4-6) (2007) 258-263.

[31] Y. Li, X. Cheng, W. Fan, Theoretic insights into the Ag doping in monolayer and bilayer $\mathrm{ZnO}$ armchair nanoribbons: edge effect and position-dependent properties, J. Nanoparticle Res. 14 (10) (2012) 1146.

[32] O.V. Bovgyra, M.V. Kovalenko, DFT Study of Electronic and Magnetic Properties of Bare and Substitutionally Doped ZnO Nanoribbons, Applied Physics (YSF), International Young Scientists Forum on, IEEE, 2015, pp. 1-4.

[33] B. Sarikavak-Lisesivdin, S.B. Lisesivdin, E. Ozbay, Ab initio study of Ru-terminated and Ru-doped armchair graphene nanoribbons, Mol. Phys. 110 (18) (2012) 2295-2300.

[34] W. Yang, R.D. Vispute, S. Choopun, R.P. Sharma, T. Venkatesan, H. Shen, Ultraviolet photoconductive detector based on epitaxial $\mathrm{Mg} 0.34 \mathrm{Zn} 0.66 \mathrm{O}$ thin films, Appl. Phys. Lett. 78 (18) (2001) 2787-2789.

[35] Version 2017.2 QuantumWise A/S. Available at: http://www.quantumwise.com.

[36] P. Narin, E. Kutlu, B. Sarikavak-Lisesivdin, S.B. Lisesivdin, E. Özbay, Electronic properties of Li-doped zigzag graphene nanoribbons, Phys. E Low-dimens. Syst. Nanostruct. 84 (2016) 543-547.
[37] Q. Chen, L. Zhu, Wang, Edge-passivation induced half-metallicity of zigzag zinc oxide nanoribbons, Appl. Phys. Lett. 95 (13) (2009) 133116.

[38] H. Q, L. Zhao, D. Pohl, J. Pang, B. Trzebicka, B. Rellinghaus, D. Pribat, T. Gemming, Z. Liu, A. Bachmatiuk, M.H. Rümmeli, Graphene-like ZnO: a mini review, Crystals 6 (2016) 1-17.

[39] X. Fan, M.L. Zhang, I. Shafiq, W.J. Zhang, C.S. Lee, S.T. Lee, ZnS/ZnO heterojunction nanoribbons, Adv. Mater. 21 (23) (2009) 2393-2396.

[40] S. Dhara, P.K. Giri, Rapid thermal annealing induced enhanced band-edge emission from ZnO nanowires, nanorods and nanoribbons, Func. Mater. Let. 4 (01) (2011) 25-29.

[41] J.C. Johnson, K.P. Knutsen, H. Yan, M. Law, Y. Zhang, P. Yang, R.J. Saykally, Ultrafast Carrier dynamics in single $\mathrm{ZnO}$ nanowire and nanoribbon lasers, Nano Lett. 4 (2) (2004) 197-204.

[42] G. Yu, C. Li, X. Ye, Electronic and magnetic properties of CdSe nanoribbon: firstprinciples calculations, Phys. Lett. 379 (1-2) (2015) 41-46.

[43] Y. Yan, S.B. Zhang, S.T. Pantelides, Control of doping by impurity chemical potentials: predictions for p-type ZnO, Phys. Rev. Lett. 86 (25) (2001) 5723-5726.

[44] H. Pan, X. Meng, D. Liu, S. Li, G. Qin, Ti/Zr, N) codoped hematite for enhancing the photoelectrochemical activity of water splitting, Phys. Chem. Chem. Phys. 17 (34) (2015) 22179-22186. 